\title{
Index
}

\section{Language Index}

Armenian 17

Baltic 273

Breton

- Middle 315-8

- Modern 308, 325, 328-9

- Old 313

British Celtic, Brittonic 240, 313

- Old Southwest Brittonic 314-5

Cornish 313-37

- Late 327-32, 335-7

- Middle 315-8, 320-9, 331-4

- Old 313

English

- Middle 15, 33, 275

- Modern 15, 22, 29, 33-4, 63, 90, 269-71, $273-6,310,312,327-32,335-6$

- Old 6, 17-8, 33, 270-1, 278, 283-4, 295,321

French

- Modern 269, 274-5, 292, 328

- Old (incl. Old Occitan) 17-8, 289, 302, $306,308,312,319-20$

Gaulish 240

German (incl. Old High German) 269-70, 273, 275-7, 292, 321

Germanic (incl. Gothic, Norse, Saxon) 17, 270, 273-4

Greek 15, 17, 25, 291, 303

Hittite 26

Icelandic 15, 33

Insular Celtic 163, 239, 242, 265, 269
Irish

- Classical Modern 49-51, 61-3, 115

- Middle 50-1, 54, 60-2, 74, 76, 78, 81-3, 85-104, 115, 117-130, 132-141, 258

- Modern 50-1, 61-7, 80-3, 189-93, 224, 269

- Old 49-83, 101, 115-32, 136, 138-9, 141, 143-177, 179-193, 195-237, 239-69, 322

Italian 269, 274

Latin $15,17,19,25,37,51,173-4,187$, 190-1, 199-200, 202-4, 215, 230, 250, $256,259,274,302-5,307-8,312$

Navajo 40

Portuguese 15, 17-8

Proto-Indo-European 70, 74, 162-4, 273

Romance 273-4, 319

Russian 17-8, 22

Sanskrit 26

Scottish Gaelic 189-93

Slavic (incl. Old Church Slavonic) 17-8, 25, 273

Spanish 17-8

Surselvan 274

Swedish 25

Welsh

- Middle 27-47, 240-1, 269-312, 320-1

- Modern 30, 42, 206, 269-71, 274-5, 295-6, 315-7, 321, 326, 335

- Old 270-1, 313-4

Ә Open Access. (c) 2020 Elliott Lash, Fangzhe Qiu, David Stifter, published by De Gruyter. (c) BY-NC-ND This work is licensed under the Creative Commons Attribution-NonCommercial-NoDerivatives 4.0 International License. 


\section{Index of Examples}

This index contains the references to all primary quotations, except those listed in the Appendix to Aaron Griffith's article (266-8)

Irish

Adomn. $2^{\text {nd }}$ Vision $\$ 21$ (Stokes 1891) 119

Airec Menman Uraird Meic Coise (Byrne 1908: 70.14) 136

Aislinge Meic Conglinne (Meyer 1892: 129.1) 140

Annals of Four Masters 899 (O'Donovan 1856) 138

Bechbretha $\S 55$ (Charles-Edwards \& Kelly 1983) 129

Blathm. 20126

Blathm. 41126

Blathm. 52118

Blathm. 108126

Blathm. 117 119-20

Blathm. 122121

Blathm. 127125

Blathm. 128120

Blathm. 140126

Blathm. 155136

Blathm. 159125

Blathm. 187125

Blathm. 190130

Blathm. 208126

Blathm. 225127

Blathm. 233125

Blathm. 237125

Blathm. 245118

Book of Armagh, Add. (Thes. 2: 240.1) 188

Book of Armagh, Add. (Thes. 2: 240.20) 187

Book of Armagh, Add. (Thes. 2: 242.20-1) 187

Book of Deer 189

Bretha Déin Chécht §37 (Binchy 1966) 129

Cambrai Homily (Thes. 2: 244.27-8) 168

Cambrai Homily (Thes. 2: 244.31) 168

Cambrai Homily (Thes. 2: 244.33) 168

Cambrai Homily (Thes. 2: 245.12) 168
Cambrai Homily (Thes. 2: 245.14) 168

Cambrai Homily (Thes. 2: 245.33) 168

Cambrai Homily (Thes. 2: 246.5-6) 168

Cambrai Homily (Thes. 2: 246.14) 167

Cambrai Homily (Thes. 2: 247.3) 168

Cambrai Homily (Thes. 2: 247.8) 210

Cambrai Homily (Thes. 2: 247.18, 39) 237

Carlsruhe Augustine $10^{\mathrm{a}} 2213$

Carlsruhe Augustine $12^{\mathrm{d}} 1210$

Carlsruhe Bede $18^{\mathrm{b}} 10228$

Carlsruhe Bede $32^{\mathrm{a}} 8118$

Carlsruhe Bede $32^{\mathrm{C}} 8125$

Carlsruhe Bede $33^{\mathrm{d}} 11$ 241-2

Carlsruhe Priscian $2^{\mathrm{a}} 3252$

Christian Brothers' Grammar (Ó hAnluain 1960 §11.36) 224

CIH 43.10132

CIH 538.19 (Senchas Már) 131-2

CIH 885.5128

$\mathrm{CIH} 920.32123$

$\mathrm{CIH} 1794.15135$

CIH 1833.30135

Coibnes Uisci Thairidni §15 (Binchy 1955) 129

Córus Bésgnai §30 (Breatnach 2017a) 129

Críth Gablach 248 (Binchy 1941) 131

Críth Gablach 280 (Binchy 1941) 131

Críth Gablach 350 (Binchy 1941) 131

Críth Gablach 448 (Binchy 1941) 131

Críth Gablach 459 (Binchy 1941) 131

Críth Gablach 593 (Binchy 1941) 131

Fél. Prol. 25-8 116

Fél. June 20116

Fél. Aug 2116

Fél. Oct 12116

Fél. Oct 16116

Fél. Oct 20116

Fél. Epil. 313117 
Fél. Epil. 411-2 116

Fo réir Choluimb céin ad.fías (Kelly 1973

§12) 322

\section{Gein Branduib maic Echach §9 (Meyer 1899) 132}

Irish Gospel of Thomas 18127 Irish Gospel of Thomas 22131 Irish Gospel of Thomas 33126 Irish Gospel of Thomas 39136 Irish Gospel of Thomas 44127 Irish Penitential (Gwynn 1914: 154-5 §1e) 126 Irish Penitential (Gwynn 1914: 160-1 §23) 127

Irish Penitential (Gwynn 1914: 166.7) 126 Irish Penitential (Gwynn 1914: 166.13) 118

LL 3565-8 (Met. Dinds.) 139 LL 3573-6 (Met. Dinds.) 139 LL 4816-9 128

LL 8367 (Táin Bó Cúailnge) 134 LL 8796 (Táin Bó Cúailnge) 134 LL 9081 (Táin Bó Cúailnge) 139 LL 12066 (Táin Bó Cúailnge) 123 LL 12726130

LL 19708 (Met. Dinds.) 139 LL 20690-1 (Met. Dinds.) 133 LL 20219 (Met. Dinds.) 120 LL 21057-8 (Prose Dinds.) 122 LL 21149 (Prose Dinds.) 120 LL 21473 (Met. Dinds.) 124 LL 22899 (Cath Ruis na Ríg) 123 LL 25163 (Met. Dinds.) 135 LL 25209 (Met. Dinds.) 135 LL 25233 (Met. Dinds.) 120 LL 25401-2 (Met. Dinds.) 134 LL 25708 (Met. Dinds.) 140 LL 25712 (Met. Dinds.) 122 LL 26022 (Met. Dinds.) 133 LL 26094 (Met. Dinds.) 133 LL 26633 (Met. Dinds.) 129 LL 26846 (Met. Dinds.) 122 LL 26995 (Met. Dinds.) 122 LL 27259-62 (Met. Dinds.) 123-4
LL 27297 (Met. Dinds.) 130

LL 27637 (Met. Dinds.) 130

LL 27775 (Met. Dinds.) 122

LL 27837 (Met. Dinds.) 122

LL 28898132

LL 29020 (Met. Dinds.) 132

LL 29245122

LL 29807 (Met. Dinds.) 134

LL 29927 (Met. Dinds.) 124

LL 30203 (Met. Dinds.) 140

LL 30502 (Met. Dinds.) 133

LL 37651 (Bórama) 135

LU 1632 (Táin Bó Flidais) 141

LU 1714 (Máel Dúin) 125

LU 1731 (Máel Dúin) 128

LU 1757 (Máel Dúin) 126

LU 1846 (Máel Dúin) 118

LU 1930 (Máel Dúin) 127

LU 2562 (Scéla na Esérgi) 133

Meyer $1903 \S 6140$

Meyer 1912 v. 23134

Ml. $16^{\mathrm{a}} 7$ 258-9, 262

MI. $17^{\mathrm{b}} 2221$

MI. $17^{\mathrm{b}} 26229$

MI. $17^{\mathrm{C}} 3$ 260-2

MI. $18^{\mathrm{a}} 7156$

Ml. $19^{\mathrm{b}} 11227,231-2$

MI. $20^{\mathrm{b}} 4122$

MI. $20^{\mathrm{d}} 11158$

Ml. $21^{\mathrm{b}} 2256$

MI. $21^{\mathrm{b}} 10156$

MI. $21^{\mathrm{d}} 4235$

MI. $22^{\text {c } 3} 159$

MI. $22^{\mathrm{C}} 14$ 206, 227, 232

MI. $22^{d} 7247$

MI. $23^{\mathrm{a}} 5187$

MI. $23^{\mathrm{a}} 12$ 257, 262

MI. $23^{\mathrm{d}} 21156$

MI. $24^{\mathrm{a}} 15156$

MI. $24^{\mathrm{a}} 18262$

Ml. $24^{\mathrm{C}} 4125$

MI. $24^{\mathrm{d}} 17262$

MI. $25^{\mathrm{C}} 16229$

MI. $25^{\mathrm{d}} 18198$

MI. $26^{\mathrm{b}} 8258$ 
MI. $26^{\mathrm{C}} 4$ 234-5

Ml. $27^{\text {d } 22 ~} 258$

MI. 28 ${ }^{\mathrm{a}} 10205$

MI. 29'13 247

MI. 29d3 156

Ml. $30^{\mathrm{a}} 3$ 202, 224

MI. $30^{\mathrm{b}} 2220$

Ml. 30 30187

MI. 31 ${ }^{\mathrm{b}} 16262$

Ml. $31^{\mathrm{b}} 22221$

Ml. $31^{\text {b } 25 ~} 258$

MI. 31'14 158, 173, 229

MI. $32^{\mathrm{a}} 5169$

MI. 32 ${ }^{d} 1203$

MI. $32^{d} 5196$

MI. $32^{d} 27158$

MI. $33^{\mathrm{a}} 1158$

MI. $33^{\mathrm{a}} 19219$

MI. $34^{\mathrm{b}} 6258$

MI. $35^{\mathrm{b}} 5215$

MI. $36^{\mathrm{b}} 1247$

MI. $36^{\mathrm{C}} 21119$

Ml. $36^{\mathrm{d}} 2221$

Ml. $37^{\text {b } 23 ~} 208$

MI. $37^{\mathrm{a}} 12258$

Ml. $37^{\mathrm{b}} 24216$

MI. $38^{\mathrm{a}} 5262$

MI. $38^{\mathrm{a}} 11156$

MI. $38^{\mathrm{C}} 3242$

MI. 38 ${ }^{\mathrm{C}} 26-7155$

Ml. 39 10246

Ml. 39 ${ }^{\mathrm{C}} 15$ 242, 244, 264-5

MI. 39' 27158

Ml. 39 28 155, 229

Ml. 39d 11 156, 221

MI. 39 13157

Ml. 39d 14229

Ml. 40 ${ }^{\mathrm{a}} 15196$

Ml. $40^{\mathrm{a}} 20184,190$

Ml. 40 22252

MI. $41^{\text {d }} 9202,225$

MI. $42^{\mathrm{b}} 18208$

MI. $42^{\mathrm{C}} 8252$

MI. $43^{\mathrm{b}} 11159$

MI. $43^{d} 14244$

MI. $44^{\mathrm{a}} 19258$

Ml. $44^{\mathrm{b}} 4$ 242, 244
MI. $44^{\mathrm{b}} 22247$

MI. $44^{\mathrm{b}} 26158$

Ml. $44^{\mathrm{C}} 15247$

MI. $44^{\mathrm{C}} 17158$

MI. 44 31159

Ml. $44^{\mathrm{C}} 32159$

MI. $45^{\mathrm{a}} 8249,251,261-2$

MI. $45^{\mathrm{a}} 16157$

Ml. $45^{\mathrm{C}} 9257,262$

MI. $46^{\mathrm{a}} 1187$

MI. $46^{\mathrm{a}} 7156$

Ml. 46 ${ }^{\mathrm{C}} 20182$

MI. $46^{\mathrm{d}} 6212$

Ml. $48^{\mathrm{d}} 6229$

MI. $48^{\mathrm{d}} 8228$

MI. $49^{\mathrm{a}} 3196$

MI. 49 11258

Ml. $50^{\text {b } 8} \quad 257,262$

MI. $51^{\mathrm{a}} 5187$

MI. $51^{\mathrm{d}} 2147$

MI. 51 ${ }^{\mathrm{d}} 12147$

MI. $51^{\mathrm{d}} 28258$

Ml. $53^{\mathrm{b}} 15157$

Ml. $53^{\mathrm{b}} 17246$

MI. $53^{\mathrm{b}} 18226$

MI. 53'13 208

MI. 53'14 159

MI. $54^{\mathrm{a}} 7$ 212, 233

MI. $54^{\mathrm{a}} 12246$

MI. $54^{\mathrm{d}} 6147$

MI. $54^{\mathrm{d}} 14159$

MI. $54^{\mathrm{d}} 23256$

MI. 55 $5^{\mathrm{c}} 1216$

MI. $55^{\mathrm{d}} 11169$

Ml. 56 ${ }^{\mathrm{a}} 20229$

Ml. $56^{\mathrm{b}} 15251$

Ml. 56 ${ }^{\mathrm{C}} 11252$

MI. 56 22205

MI. $57^{\mathrm{a}} 10230$

MI. 57 ${ }^{\mathrm{C}} 12$ 119, 218, 230-1, 235

MI. $58^{\mathrm{d}} 14156$

MI. 59a 12 260, 262

MI. $59^{\mathrm{a}} 21156$

Ml. 59 $9^{\text {b }}$ 230-1

Ml. 59 12157

MI. $61^{\mathrm{a}} 21202-3$

MI. $61^{\mathrm{a}} 22$ 202-3 
Ml. $61^{\mathrm{b}} 7125$

MI. $61^{\mathrm{b}} 17244$

MI. $62^{\mathrm{a}} 2252$

MI. $62^{\mathrm{C}} 7257$

MI. $62^{\mathrm{C}} 16158$

MI. $62^{\mathrm{C}} 21158$

MI. $63^{\mathrm{b}} 1159$

Ml. $63^{\mathrm{b}} 9213$

MI. $64^{\mathrm{a}} 12259$

Ml. $64^{\mathrm{a}} 13$ 248, 259, 262

MI. $65^{\mathrm{a}} 12122$

ML. $66^{\mathrm{d}} 14205$

MI. $68^{\mathrm{d}} 15247$

MI. $69^{\mathrm{a}} 17196$

MI. $69^{\mathrm{a}} 18244$

MI. $69^{\mathrm{a}} 23188$

MI. $70^{\mathrm{a}} 12229$

Ml. 70'6 125

MI. $71^{\mathrm{c}} 9252$

MI. $72^{\mathrm{C}} 4122$

MI. $72^{\mathrm{C}} 9187$

MI. $73^{\mathrm{C}} 2231$

MI. $74^{\mathrm{b}} 22148$

MI. $74^{d} 3258$

MI. $74^{\mathrm{d}} 7198$

MI. $75^{\mathrm{b}} 7119$

MI. $75^{\mathrm{b}} 7209$

MI. $75^{\mathrm{C}} 8122$

MI. $76^{\mathrm{a}} 12246$

MI. $79^{\mathrm{b}} 5211$

Ml. $84^{\mathrm{c}} 9258$

MI. $85^{\mathrm{b}} 11219$

MI. $85^{\mathrm{d}} 10230$

MI. 86 3125

MI. 86 ${ }^{\mathrm{C}} 10157$

MI. $86^{\text {d } 6} 209$

MI. $86^{\mathrm{d}} 19 \mathrm{a} 242,245,263,265$

MI. $87^{\mathrm{a}} 8$ 208, 233

MI. $90^{\mathrm{b}} 10119$

ML. $92^{\mathrm{b}} 9$ 209, 232

MI. $93^{\mathrm{a}} 13169$

MI. $93^{\mathrm{a}} 15159$

MI. $93^{d} 12157$

MI. $93^{\mathrm{d}} 14221$

MI. 95² 213-4

MI. $96^{\mathrm{a}} 13187$

MI. $96^{\mathrm{b}} 13122$
MI. $96^{\mathrm{C}} 11258$

MI. $97^{\mathrm{a}} 7243$

MI. $98^{\mathrm{d}} 8246$

MI. $100^{\mathrm{a}} 10200$

MI. $104^{\mathrm{a}} 4125$

MI. $104^{\mathrm{b}} 5212$

MI. $106^{\mathrm{b}} 8174$

MI. $108^{\mathrm{a}} 9229$

Ml. $109^{\mathrm{b}} 2171$

MI. $110^{\mathrm{a}} 10205$

MI. $110^{\mathrm{d}} 10187$

MI. $111^{\mathrm{b}} 15252$

MI. $111^{\mathrm{b}} 17213$

MI. $111^{d} 4209$

MI. $112^{\mathrm{b}} 12210$

MI. $114^{\mathrm{a}} 7$ 156, 221

MI. $114^{\mathrm{a}} 9257$

MI. $114^{\mathrm{C}} 3256$

MI. $114^{\mathrm{d}} 10158$

Ml. $115^{\mathrm{b}} 15$ 251-2

MI. $115^{\mathrm{c}} 1125$

MI. $115^{\text {d }} 9214$

MI. $117^{\mathrm{b}} 9256$

Ml. $120^{\mathrm{a}} 3159$

MI. $120^{\mathrm{a}} 6256$

MI. $120^{\mathrm{d}} 2258$

MI. $121^{\mathrm{d}} 22158$

MI. $122^{\mathrm{C}} 9125$

MI. $123^{\mathrm{b}} 7199$

MI. $124^{\text {b }} 3147,242,244$

MI. $124^{\mathrm{d}} 7221$

Ml. $125^{\mathrm{a}} 9156$

MI. $125^{\mathrm{d}} 4242$

MI. $126^{\mathrm{b}} 12246$

MI. $127^{\mathrm{C}} 8157$

MI. $127^{\mathrm{d}} 8184$

MI. $129^{\mathrm{b}} 1213,230$

MI. $129^{\mathrm{b}} 2$ 227-8, 230

MI. $130^{\mathrm{a}} 16125$

MI. $131^{\mathrm{b}} 8229$

MI. $131^{\mathrm{C}} 10$ 154, 172

MI. $131^{\mathrm{d}} 12$ 119, 154

MI. $134^{\mathrm{a}} 1156$

MI. $135^{\mathrm{a}} 13209$

MI. $135^{\mathrm{d}} 4159$

MI. 136' 11201

Murphy 1956: 26-7 128 
O’Brien 1952, verse 12c 132

ó Cuív 1966: 174140

Olr. Homily (Strachan 1907: 3) 222

Olr. Table of Commutations §12 (Binchy 1962) 118

Passions and Homilies from Leabhar Breac (Atkinson 1887: 643) 133

RIA MS 23 P 2, f. $222^{\text {ra } 10} 137$

Saltair na Rann 1306137

Saltair na Rann 1942137

Saltair na Rann 5367124

Scél Mongáin (White 2006: 76) 118

Sg. $2^{\mathrm{b}} 2190$

Sg. $3^{\mathrm{a}} 1247$

Sg. $4^{\mathrm{b}} 12125$

Sg. $6^{\mathrm{b}} 11188$

Sg. $8^{\mathrm{b}} 2256$

Sg. $9^{\mathrm{a}} 16256$

Sg. $17^{\mathrm{a}} 7156$

Sg. $26^{\mathrm{a}} 5215$

Sg. $26^{\mathrm{a}} 8215$

Sg. $26^{\mathrm{b}} 12183$

Sg. $28^{\mathrm{a}} 4256$

Sg. $29^{\mathrm{a}} 3255$

Sg. $33^{\mathrm{a}} 17256$

Sg. 33 ${ }^{\mathrm{a}} 19 \mathrm{a} 191$

Sg. $33^{\mathrm{a}} 23256$

Sg. $36^{\mathrm{b}} 1256$

Sg. $39^{\mathrm{b}} 1150,256$

Sg. $39^{\mathrm{a}} 25215$

Sg. $40^{\mathrm{a}} 15229$

Sg. $45^{\mathrm{a}} 15214$

Sg. $45^{\mathrm{b}} 19$ 190, 251-2

Sg. $50^{\mathrm{a}} 1256$

Sg. $51^{\mathrm{a}} 4256$

Sg. $53^{\mathrm{a}} 11256$

Sg. $56^{\mathrm{b}} 10256$

Sg. $59^{\mathrm{b}} 12256$

Sg. $61^{\mathrm{a}} 1256$

Sg. $62^{\mathrm{b}} 2204$

Sg. $63^{\mathrm{a}} 16184$

Sg. $70^{\mathrm{a}} 6215$

Sg. $73^{\mathrm{b}} 1191$
Sg. $77^{b_{5}} 252$

Sg. $90^{\mathrm{b}} 12191$

Sg. $104^{\mathrm{b}} 1125$

Sg. $137^{\text {b } 2 ~} 208$

Sg. $139^{\mathrm{b}} 2159$

Sg. $145^{\mathrm{a}} 4213$

Sg. $146^{\mathrm{b}} 16255$

Sg. $148^{\mathrm{b}} 12125$

Sg. $150^{\mathrm{b}} 1121$

Sg. $150^{\mathrm{b}} 5121$

Sg. $161^{\mathrm{a}} 1252$

Sg. $180^{\text {b } 2} 255$

Sg. $183^{\mathrm{a}} 3159$

Sg. $183^{\mathrm{b}} 3220$

Sg. $188^{\mathrm{a}} 7256$

Sg. $188^{\mathrm{a}} 8256$

Sg. $188^{\mathrm{a}} 12256$

Sg. $188^{\mathrm{a}} 13256$

Sg. $188^{\mathrm{a}} 16256$

Sg. $188^{\mathrm{a}} 19256$

Sg. $193^{\mathrm{a}} 1256$

Sg. $197^{\mathrm{a}} 11$ 210-11

Sg. $197^{\mathrm{b}} 4255$

Sg. $198^{\text {b }} 3255$

Sg. $200^{\mathrm{b}} 6187$

Sg. $201^{\mathrm{b}} 6190$

Sg. $202^{\mathrm{b}} 3235$

Sg. $203^{\mathrm{a}} 16125$

Sg. $208^{\mathrm{b}} 15224$

Sg. $209^{\mathrm{b}} 10187$

Sg. $209^{\mathrm{b}} 13255$

Sg. $210^{\mathrm{a}} 10255$

Sg. $215^{\mathrm{a}} 2183$

Sg. $218^{\mathrm{a}} 9256$

Sg. $220^{\mathrm{a}} 6215$

Sg. $221^{\text {b }} 7229$

Southampt. Psalter (Ó Néill 2012: LXIII no. 12) 117

Táin Bó Fraích (Meid 1974: 130-1) 260

Thes. 2: 241.15131

Thes. 2: 241.16242

Thes. 2: 241.8-9 228, 235-6

Thes. 2: 252.14117

Thes. 2: 253.16117

Thes. 2: 255.7117 
Thes. 2: 290.14119

Thes. 2: 292 v.8 119

Thes. 2: 294.13119

Thes. 2: 312.4 217-8, 222

Thes. 2: 317.6 205-6

Tochmarc Étaíne (Bergin \& Best 1934-8 §23) 221

Tur. $58^{\mathrm{a}} 234$

Tur. 60242

Tur. 108183

Vienna Bede 23183

Vienna Bede 31183

Vita Tripartita (Mulchrone 1939: I. 116;

Stokes 1887: 11) 138

Vita Tripartita (Mulchrone 1939: I. 2417;

Stokes 1887: 207) 138-9

Wb. $1^{\mathrm{a}} 2153$

Wb. $1^{\mathrm{b}} 14198$

Wb. $1^{\mathrm{c}} 20203$

Wb. $2^{\mathrm{a}} 4203$

Wb. $2^{\mathrm{a}} 11226$

Wb. $2^{\mathrm{a}} 12156$

Wb. $3^{\mathrm{a}} 15262$

Wb. $3^{\mathrm{b}} 1251$

Wb. $3^{\mathrm{b}} 10236$

Wb. $3^{\text {b } 23} 159$

Wb. $3^{\mathrm{c}} 21251$

Wb. $3^{\mathrm{c}} 25226$

Wb. 3'33 169

Wb. 3`34 169

Wb. $3^{\mathrm{d}} 10196$

Wb. $3^{\mathrm{d}} 27228$

Wb. $4^{\mathrm{b}} 19226$

Wb. 4 ${ }^{\mathrm{c}} 13226$

Wb. $4^{\mathrm{c}} 27$ 216-8, 222, 227

Wb. $4^{\mathrm{c} 32} 207,211,232-3$

Wb. $4^{\mathrm{c}} 33$ 195, 207, 211, 232-3

Wb. $4^{\mathrm{d}} 32244$

Wb. $5^{\mathrm{b}} 12251$

Wb. $5^{\text {b }} 28$ 184, 219, 222

Wb. $5^{\mathrm{b}} 29254$

Wb. $5^{\mathrm{b}} 30157$

Wb. $5^{\text {b } 32} 217-8,222,227$

Wb. $5^{\mathrm{b}} 35163$

Wb. $5^{\mathrm{c}} 6153,232$
Wb. 5'16 181

Wb. $5^{\mathrm{c}} 23226$

Wb. $5^{d} 5169$

Wb. $6^{\mathrm{a}} 13229$

Wb. $6^{\text {c }} 8$ 201, 227, 250

Wb. $6^{\mathrm{b}} 13157$

Wb. $6^{\mathrm{b}} 22226$

Wb. $6^{\mathrm{d}} 11154$

Wb. $7^{\mathrm{a}} 2252$

Wb. $7^{\mathrm{a}} 4158$

Wb. $7^{\mathrm{a}} 5158$

Wb. $7^{\mathrm{a}} 12$ 159, 181

Wb. $7^{\mathrm{b}} 11240,242$

Wb. $7^{\mathrm{b}} 15232$

Wb. $7^{\mathrm{d}} 3181$

Wb. 8'16 157

Wb. $9^{\mathrm{a}} 16159$

Wb. $9^{\mathrm{a}} 23156$

Wb. $9^{\text {b } 3} 226$

Wb. $9^{\mathrm{b}} 13226$

Wb. $9^{\mathrm{b}} 19156$

Wb. $9^{\mathrm{C}} 10$ 226, 228

Wb. 9'20 157

Wb. $9^{d} 6204$

Wb. $9^{\mathrm{d}} 9198$

Wb. $10^{\text {b } 3} 182,226$

Wb. $10^{\mathrm{b}} 27122$

Wb. $10^{\mathrm{C}} 1182$

Wb. 10' 22237

Wb. $10^{\mathrm{d}} 26157,220$

Wb. $11^{\mathrm{a}} 19226$

Wb. $11^{\mathrm{d}} 12181$

Wb. $12^{\mathrm{c}} 5200$

Wb. $12^{\mathrm{C}} 8247$

Wb. $13^{\mathrm{a}} 29$ 217-8, 227, 231-2

Wb. $13^{\mathrm{b}} 7215$

Wb. $13^{\mathrm{c}} 24226$

Wb. $13^{\mathrm{d}} 20211$

Wb. $14^{\mathrm{a}} 29242$

Wb. $14^{\mathrm{b}} 13226$

Wb. $14^{\mathrm{c}} 18$ 58, 154

Wb. $14^{\mathrm{c}} 20157$

Wb. $14^{\mathrm{C}} 42169$

Wb. $14^{\mathrm{d}} 37157$

Wb. $15^{\mathrm{d}} 12181$

Wb. $16^{\mathrm{a}} 2226$

Wb. $16^{\mathrm{a}} 11211$ 


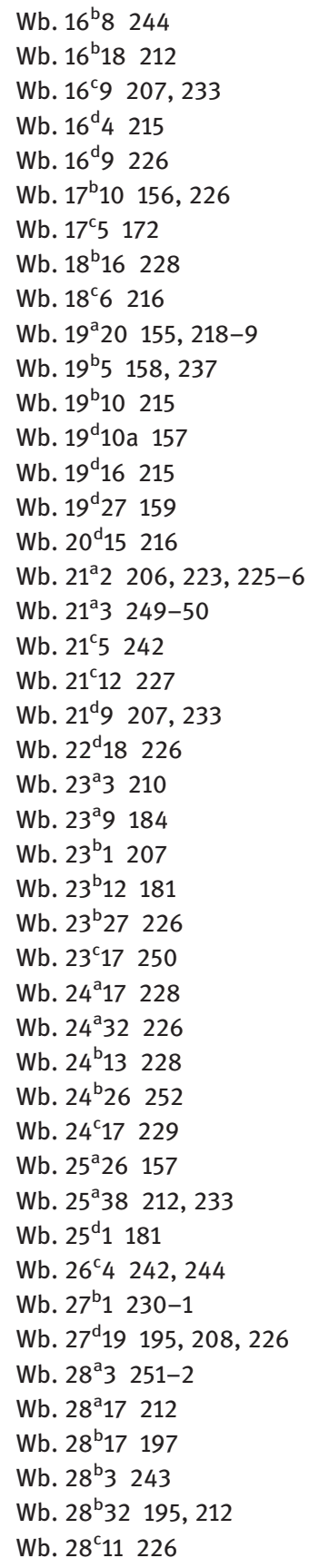

Wb. $28^{\mathrm{C}} 14199$

Wb. $29^{\mathrm{a}} 25163$

Wb. $29^{\mathrm{C}} 4$ 209, 224

Wb. $29^{\mathrm{d}} 29254$

Wb. $30^{\text {a } 9} 156$

Wb. $30^{\mathrm{d}} 13226$

Wb. $31^{\mathrm{a}} 6223$

Wb. $31^{\mathrm{C}} 11228$

Wb. $31^{\mathrm{C}} 16159,250$

Wb. $31^{\mathrm{c}} 17226$

Wb. $32^{\mathrm{a}} 25$ 201-2

Wb. $32^{\mathrm{C}} 10230$

Wb. $33^{\mathrm{a}} 12158$

Wb. $33^{\text {b } 1} 228,231,237$

Wb. $33^{\text {b } 13} 169$

Wb. $33^{\mathrm{C}} 12169$

Latin

2 Cor. 7:13 212

Historia Regum Britanniae, Geoffrey of Monmouth (Reeve \& Wright 2007: 45 §32) 303

Imago Mundi (Flint 1983; 48-9) 307

Mt. 27:42 299

Philemon 21201

Pilgrim Girardus, BL Arundel MS 346 (Neuhaus 1886: 38) 305

Pilgrim Girardus, BL Cotton Cleopatra MS C X (Neuhaus 1886: 38) 305-6

Pilgrim Girardus, BL Cotton Cleopatra MS C X (Neuhaus 1886: 39) 306

Rom. 3:2 203

1 Tim. 3:16-4:1 199-200

\section{English}

Ælfric, Genesis 3.8271

Elfric, Grammar 96.11-2 271

Genesis 2629284

Orosius IV.11 271

Wycliffe, Old Testament, Genesis 3.8272

\section{French}

Boeve de Haumtone (Stimming 1899: 68,

$$
\text { I. 1797) } 307
$$


Queste del Saint Graal (Pauphilet 1923

l. 129) 319

Vie de sainte Doucline (Albanès 1879

§41) 320

Southwest British (Cornish and Breton)

Angers MS 477 fol. 62b (Fleuriot 1964b:

413) 314

Angers MS 477 fol. 65a (Fleuriot 1964b:

262) 314

Angers MS 477 fol. 79a (Fleuriot 1964b:

412) 314

Beunans Meriasek l. 594317

Beunans Meriasek I. 941318

Beunans Meriasek I. 970-77 324

Beunans Meriasek l. 978323

Beunans Meriasek I. 978-82 323

Beunans Meriasek I. 1801-8 334

Beunans Meriasek I. 1807-8 332-4

Beunans Meriasek l. 1888 332-4

Beunans Meriasek I. 3224-5 332-4

Beunans Meriasek I. 4340-2 332-4

Beunans Meriasek I. 4515-6 332-4

Bewnans Ke (Thomas \& Williams 2007)

II. 208-12) 321

Bewnans Ke (Thomas \& Williams 2007)

II. 213) 321

Bible translation of Wella Rowe (Loth 1902: 180) 330

Boson family, writings (Padel 1975 §3) 330

Boson family, writings (Padel 1975 §46) 330

Middle Cornish Charter (Toorians 1991

I. 15-22) 322

Middle Cornish Charter (Toorians 1991

I. 20) 321

Norris 1859, 1: I. 1259-64 323

Norris 1859, 1: I. 1263321

Norris 1859, 1: l. 2423-6 325

Norris 1859, 2: l. 909317

Passion (Stokes 1860-1: st. $9^{\mathrm{a}}$ ) 317

Passion (Stokes 1860-1: st. 42 ${ }^{\mathrm{a}}$ ) 325

Passion (Stokes 1860-1: st. 54 ${ }^{\mathrm{a}}$ ) 325

Tregear Homilies $1^{r}$, II. 1-5 335-6

Tregear Homilies $1^{\text {r }}$, I. 3328

Tregear Homilies 1', I. 5328

Tregear Homilies $2^{r}$, Il. 18-9 328
Tregear Homilies $4^{r}$, II. 2-3 328-9

Tregear Homilies $4^{r}$, II. 2-6 336-7

Tregear Homilies 8v, I. 14328

Vie de sainte Catherine (Ernault 1887a

§8) 316

Vie de sainte Catherine (Ernault 1887a

§12) 316

Vie de sainte Catherine (Ernault 1887a

§13) 316

Vie de sainte Nonne (Ernault 1887b

l. 240) 318

William Bodinar's letter (Pool \& Padel

1976-6: 234.7) 331

Welsh

Breuddwyd Maxen (Williams 1908 Il. 18-9) 44

Brut y Brenhinedd (Roberts 1971 II. 795-7) 320

Brut y Brenhinoedd, BL Cotton Cleopatra MS B V i, 2v: 7-9 282-3

Brut y Brenhinoedd, BL Cotton Cleopatra MS B V i, 5: 27293

Brut y Brenhinoedd, BL Cotton Cleopatra MS B V i, 6r: 10-11 293-4

Brut y Brenhinoedd, BL Cotton Cleopatra MS B V i, $11^{\mathrm{r}}: 9286$

Brut y Brenhinoedd, BL Cotton Cleopatra MS B V i, 11': 21285

Brut y Brenhinoedd, BL Cotton Cleopatra MS B V i, $11^{\mathrm{v}}: 3283$

Brut y Brenhinoedd, BL Cotton Cleopatra MS B V i, 19v : 1 285-6

Brut y Brenhinoedd, BL Cotton Cleopatra MS B V i, 20 ${ }^{r}$ : 14-6 303-4

Brut y Brenhinoedd, BL Cotton Cleopatra MS B V i, 24v 15283

Brut y Brenhinoedd, BL Cotton Cleopatra MS B V i, $34^{\mathrm{v}}: 23283$

Brut y Brenhinoedd, BL Cotton Cleopatra MS B V i, $55^{\mathrm{v}}: 19293$

Brut y Brenhinoedd, BL Cotton Cleopatra MS B V i, 96 ${ }^{\mathrm{v}}:$ 22-3 295

Brut y Brenhinoedd, Cardiff MS 1.362 (Hafod 1), $63^{\mathrm{v}}: 19284$

Brut y Brenhinoedd, NLW MS 3035, 61: 13281 
Brut y Brenhinoedd, NLW MS Peniarth 46, 254: 16281

Brut y Brenhinoedd, Oxford Jesus Coll. MS 111, 39 (154): 27302

Credo Athanasius (Intr.), NLW MS Peniarth 5 , 14g. B v. 196301

Culhwch ac Olwen (Bromwich \& Evans 1992 l. 595) 38

Cynghorau Catwn (Lewis 1935: 23.28) 277

Cynghorau Catwn (Lewis 1935: 24.25) 277

Cynghorau Catwn (Lewis 1935: 29.37) 277

Cynghorau Catwn, NLW MS Llanstephan 27, 31: 3309

Cynghorau Catwn, NLW MS Llanstephan 27, 32: 20309

Cynghorau Catwn, NLW MS Llanstephan 27, $165^{\mathrm{v}}: 15310$

Cynghorau Catwn, NLW MS Llanstephan 27, $168^{\mathrm{r}}: 16290,308$

Cynghorau Catwn, NLW MS Peniarth 3 ii, 37: 3 308-9

Cynghorau Catwn, NLW MS Peniarth 3 ii, 38 : 11290

Delw'r Byd, Oxford Jesus Coll. 111, $243^{r}$ (976): 14 307-8

De Mensuris et Ponderibus 85 (Lambert 2003: 113) 314

Efengyl Nicodemus, NLW MS Peniarth 5, 32 ${ }^{\mathrm{r}}$ : 14300

Gwyrtheu Mair, NLW MS Llanstephan 27, $176^{\mathrm{r}}: 10301$

Gwyrtheu Mair, NLW MS Peniarth 14 Jones 1939: 148) 301

Gwyrtheu Mair, NLW MS Peniarth 14 (Jones 1941: 24) 300, 305-6

Gwyrtheu Mair, NLW MS Peniarth 14 Jones 1941: 25) 300, 306

Gwyrtheu Mair (Jones 1939: 336.33) 276 Juvencus Englynion $5^{\mathrm{a}-\mathrm{b}} 314$

NLW MS Peniarth 9, 1: 4302

Oxford Jesus College MS 111, $95^{\vee}$ (400): 9302

Pedeir Keinc y Mabinogi (Williams 1951: 1. 3-4) 38
Pedeir Keinc y Mabinogi (Williams 1951: 2.

12-13) 277

Pedeir Keinc y Mabinogi (Williams 1951: 16. 18-9) 316

Pedeir Keinc y Mabinogi (Williams 1951: 20.29) 276

Pedeir Keinc y Mabinogi (Williams 1951: 44.11) 316

Pedeir Keinc y Mabinogi (Williams 1951: 45. 2-3) 316

Pedeir Keinc y Mabinogi (Williams 1951: 46.27) 276

Pedeir Keinc y Mabinogi (Williams 1951: 68. 15-6) 317

Poppe 2009: 254296

Saith Doethion Rhufain, Oxford Jesus Coll. MS 20, $56^{\text {av }}: 1295$

Saith Doethion Rhufain, Oxford Jesus Coll. MS 111, 131' (541): 34294

'Surexit' Memorandum 313

Thomas 1996 (Grammar), 269295

Y Groglith, NLW MS Llanstephan 27, 105: 17299

Y Groglith, NLW MS Peniarth 5, 7v : 18309

$Y$ Groglith, NLW MS Peniarth 5, $7^{\mathrm{v}}: 20300$

$Y$ Groglith, NLW MS Peniarth 7, 58 ${ }^{v}$ (215): 2299

Y Groglith, Shrewsbury MS 11, 113: 16299

Y Groglith, Shrewsbury MS 11, 114: 1309

Ystoria Bilatus, NLW MS Peniarth 5, $11^{\mathrm{r}}$ : 14 303-4

Ystoria Bilatus, NLW MS Peniarth 7, $63^{\mathrm{v}}$ (236): 20 303-4

Ystoria Bown de Hamtwn, NLW MS Peniarth 5, 134 (301): 22307

Ystoria Bown de Hamtwn, NLW MS Peniarth 5, $148^{v} 292$

Ystoria Carolo Magno: Chronicl Turpin, NLW MS Peniarth 5, $74^{r}$ (63): 34302

Ystoria Titus, NLW MS Peniarth 5, $37^{\mathrm{r}}$ : 48 303-4

Ystoriau Saint Greal, NLW MS Peniarth 11, $63^{\mathrm{v}}: 24294$

Ystoriau Saint Greal, NLW MS Peniarth 11, $239^{\mathrm{v}}: 9287$ 


\section{Thematic Index}

absolute / conjunct distinction 239-40, 265 adjectives 36

- comparison 36, 119, 121, 206-11, 223-4, 284

- positive 211-6

adverbs, adverbials $36,38,152,172$, 179-80, 195-204, 206, 214-5, 223, 225, 228-9, 248-9, 257-8, 263, 269, 272-3, $284,315-8,325$

antecedent 250

- adjectival 195-237

- adverbial 197, 202, 225, 228-9, 249

- mixed 204, 224-5, 233

- prepositional 239, 249-50, 253-63

- subject/object 196, 200-2, 218, 220, 222-3, 227, 233

authorship attribution 85-87, 89-90, 97, 99, 101-4

clause types $143-8,151-4,163-73,175-7$, 313

cleft sentence 44, 152-4, 195-206, 215-9, 222, 224-5, 228-36, 239, 245, 248-60, 263-6, 315, 317

complement clause $36,172,198$

complexity of language $24-5,49-50,59$, $65-66,69,72$

configuration/constituent order 17, 19 , 44-5, 47, 152, 313-32

contracted verbs $251-2$

convergence $270,302,308,312$

corpora, treebanks and texts 15-26, 33, 45-7

- dictionaries 19, 22, 49, 61-5, 70, 79-80, 83

- Irish 1-3, 63-4, 67, 78, 85-9, 94-5, 101-2

- Late Cornish 327-32

- Middle Cornish 325-7, 332

- Welsh 27-9, 46-7, 272, 278-9, 281-2, 286-90, 296-7, 311

deictic particle $130,136-7$

dialects $188-92$ figura etymologica 153, 196-7, 220, 248

finite-state morphology/transducer 21 , $23-4,66-73,75-6,78-83$

information structure $17-8,20,29-30,33$, $36,41,44-5,47$

intensifier 269-78, 280-5, 287, 293-7, 299, 303-5, 307, 309-11

left periphery $314-5,317-9,321-2,324,334$

machine learning $24,66,79,85,87,89,94$, 103-4

morphological analyser $23,49-50,65-8$, 82-3

morphosyntax, morphosyntactic annotation 15, 17, 19, 22-3, 28, 31-3, $37,40-1,47,54,65,154$

mutations $28-9,32-3,40,56,90,92$, $145-6,189,239,250$

- lenition 252

- nasalisation 56, 58, 120, 145-6, 152-3, 165-7, 179-93, 195-8, 202-3, 205-25, 227-33, 237, 250, 252, 256-8, 261-3

natural language processing (NLP), toolkit 22, 28, 31, 33, 41, 50, 61, 64, 66

NLP pipeline $29-45,64$

- annotation 15-47, 63-4

- lemmatisation/lemmatiser 16, 19, 25 , 64-7, 71, 79-82

- POS tagging/tagger 15-6, 21-40, 44-7, 64-8, 75, 81-3, 89-90, 101, 104

- pre-processing 29-32

- syntactic parsing/parser 15-6, 21-2, 25-31, 35-7, 41, 43-7, 52, 62, 64, 67-8, $70,75-6,78-9,332$

- tokenisation 26, 31-2, 47, 64

nota augens $56-60,115-6$

orthography $25,28,31-3,40,64,71,82,92$, 115, 250, 329 
- standardisation, normalisation 19, 24-5, 28-30, 33, 47, 50-1, 64-7, 71, 79-82, 90, 92-3, 104, 189

paradigmatic split $173-5$

particle theory/'Cowgill particle' 239-40, 246-8, 263-5

phonological changes

- depalatalisation 166-9

- i-apocope 239-40

- loss of nasals 189, 192, 196, 229

- pretonic e > a 242

preverbs $37-8,52-8,60,70-2,76-8,80-1$, $143-5,147-51,155-71,174-6,214$, 220-1, 225-6, 231, 239-8, 261, 263-5, 308,310

pronouns $34-36$

- demonstrative, Old Irish 115-141, 153

- infixed (general) 56, 86, 90, 119, 246, 261, 271, 288, 294-5, 309-11

- infixed, Class A 56, 143-55, 162-7, 169, $172,175-6,216-9,221,226-7,229-31$, 233, 244, 250

- infixed, Class B 56, 143-67, 169-70, 172-7, 216-8, 220-2, 229, 233, 244, 250

- infixed, Class C 56, 143-7, 149, 151-67, 169-76, 218-22, 227, 230-1, 233, 250

- prepositions with pronominal objects $34-35,38,90,121,127-8,134$, 166-8, 179-180, 182-3, 187, 191, 243, 281, 283-5 reflexive pronouns/markers, reflexivity $269-78,281-2,286-312$ relative clauses $58,143-7,151-3,162-3$, 165-7, 169-72, 175, 195-237, 248-262

- leniting relative clauses $166,169-70,196$, 201, 218-23, 225, 232-3, 237, 250, 252, 254, 256, 258-61, 263

- nasalising relative clauses $58,152-3$, 195-233, 237, 249, 252, 256-8, 262-3

relative marker $76,78,174,230$

statistical methods $22,24,65-6,85-6$, 89-93, 99-100

- Fisher's exact test 241

- F-scores 65

- k-medoids 91-104

- Principal Component Analysis 93-4

- ten-fold cross-validation 39

syntactic raising 206, 223-4, 228, 231, 233, 237

syntax, syntactic annotation 16-21, 23-4, 29-31, 36, 41-3, 45-7, 64, 143, 146, 152-6, 160-1, 171-2, 176, 179-80, 183-6, 293-7, 308, 313-32

verbal complex 52-60, 70-1, 75, 143, $145-55,166,168-9,171,176,223$, 243-5, 248

XML, TEI XML 20, 30, 43, 46-7, 63, 86, 88-9 


\section{Word Index}

\section{Old and Middle Irish}

$a^{N}$ 'when' 228-9

ad- 147, 151, 160, 220

aisndis, aisndisse 186

aith- 163-4

améin/amné 258, 263

ar-, ara $168,226,233,244$

arindí 257

as-, asa $147,244,255$

as.beir, epir 73-4

as. oilgi 70

at.tá $234-7$

beirid 75

bete 237

ceta-, cita-, ciata- 'first' 239-48, 263-5

ceta- 'along' 241, 246-8, 264-5

cían 205-6, 225

Co $^{H} 215$

con- 151

conid. 174

cruth, in chruth 228-9, 249

$-d^{L}-152$

de/i- 150

di.sruthaigedar, dissruthigthe 255-6

do- 150

dó 252

do.esta 169

do·léici, ·teilci, ·tarlaic 71-3, 76-7

dommae 236

etar, itar 242-4

fo.fera 169-71, 173, 176

forngaire 186

frecndairc, frecndarcus 186

fris- 160, 174-5, 221

frit-racatar 172

i $130,136-7$

imm-, imma. 244

immaircide 258-9

in(d)-, inda. (preverb) 244

in(d) (adverbial particle) 214-5

léicid 68, 73-4, 76-7

ocu- 246-8, 264

ol sé, ol sí 137 -s (infixed pronoun as relative marker) 230

samlaid 258, 263

sé, sí (personal pronoun) 137-41

sin, sen, sein, sain 115, 117-24, 131-4, 136-7, 141

$-\sin 115-6$

síu 128, 130, 136

só, sé, séo (demonstrative pronoun) 115, 117, 124-31, 134-5, 138-40

-so, -se $115-7$

sommae $236-7$

sund 129

túailnge, túailngigidir 186

\section{Welsh}

efo $280-1$

hun(an) 269-70, 276-312

sef 30

ym- 270-1, 274-7, 286-93, 296-7, 300-2, $307,310-1$

ynteu 280

\section{Breton}

em- 308

en, em 308

Latin

deus 173-4

dīuus 173-4

ipse 305-7

se $274,291,303-4,306-7$

te $304-5$

\section{Proto-Celtic}

*ambi 276

*enter 242-3

*eti 240, 246, 263

*kanta 241, 247, 264

*kentu- 240, 242-8, 263

*onku- 246, 248, 264

Proto-Indo-European

${ }^{*} s(u) e-269-70,274$ 


\section{Germanic}

hine, hem, him (Old and Middle

English) 271-2, 284

selbst (German) 269, 273, 276-7, 310-1

-self (English) 269-74, 276, 284, 310-1

ser, sik (Old Norse) 270

sich (German) 269, 273, 275, 277, 292, 311

sih (Old High German) 270 sik (Old Saxon) 270

sik, sis (Gothic) 270

\section{Romance}

me (Old French) 307

se (French) 269, 274-5, 308

se- (Surselvan) 274

si (Italian) 269, 274 